\title{
Semiclassical theory of shot-noise suppression
}

\author{
M. J. M. de Jong \\ Philips Research Laboratories, 5656 AA Eindhoven, The Netherlands \\ and Instituut-Lorentz, University of Leiden, 2300 RA Leiden, The Netherlands \\ C. W. J. Beenakker \\ Instituut-Lorentz, University of Leiden, 2300 RA Leiden, The Netherlands
}

(Received 13 December 1994)

\begin{abstract}
The Boltzmann-Langevin equation is used to relate the shot-noise power of a mesoscopic conductor to classical transmission probabilities at the Fermi level. This semiclassical theory is applied to tunneling through $n$ barriers in series. For $n \rightarrow \infty$ the shot noise approaches one-third of the Poisson noise, independent of the transparency of the barriers. This confirms that the one-third suppression known to occur in diffusive conductors does not require phase coherence.
\end{abstract}

The discreteness of the electron charge causes timedependent fluctuations in the electrical current, known as shot noise. These fluctuations are characterized by a white noise spectrum and persist down to zero temperature. The shot-noise power $P$ contains information on the conduction process, which is not given by the resistance. A well-known example is a vacuum diode, where $P=2 e|I| \equiv P_{\text {Poisson, }}$ with $I$ the average current. This tells us that the electrons traverse the conductor in a completely uncorrelated fashion, as in a Poisson process. In macroscopic samples, the shot noise is averaged out to zero by inelastic scattering.

In the past few years, the shot noise has been investigated in mesoscopic conductors, smaller than the inelastic scattering length. Theoretical analysis shows that the shot noise can be suppressed below $P_{\text {Poisson, due to }}$ correlations in the electron transmission imposed by the Pauli principle. ${ }^{1-5}$ Most intriguingly, it has been found that $P=\frac{1}{3} P_{\text {Poisson }}$ in a metallic, diffusive conductor. ${ }^{6-10}$ The factor one-third is universal in the sense that it is independent of the material, sample size, or degree of disorder, as long as the length $L$ of the conductor is greater than the mean free path $\ell$ and shorter than the localization length. An observation of suppressed shot noise in a diffusive conductor has been reported. ${ }^{11}$ In a quantummechanical description, ${ }^{6}$ the suppression follows from the bimodal distribution of transmission eigenvalues. ${ }^{12}$ Surprisingly, Nagaev ${ }^{7}$ finds the same one-third suppression from a semiclassical approach, in which the Pauli principle is accounted for, but the motion of electrons is treated classically. This implies that phase coherence is not essential for the suppression, but the relationship between the quantum-mechanical and semiclassical theories remains unclear.

In this paper, we reinvestigate the semiclassical approach and present a detailed comparison with quantummechanical calculations in the literature. We use the Boltzmann-Langevin equation, ${ }^{13,14}$ which is a semiclassical kinetic equation for nonequilibrium fluctuations. This equation has previously been applied to shot noise by Kulik and Omel'yanchuk ${ }^{15}$ for a ballistic point contact, and by Nagaev ${ }^{7}$ for a diffusive conductor. Below. we will demonstrate how the Boltzmann-Langevin equation can be applied to an arbitrary mesoscopic conductor. Our analysis corrects a previous paper. ${ }^{16}$ To be specific, we consider tunneling through $n$ planar barriers in series (tunnel probability $\Gamma$ ). This model is sufficiently simple that it allows us to obtain a closed expression for $P$ and sufficiently general so that we can compare it with all results in the literature from the quantum-mechanical approach. For $n=2$ and $\Gamma \ll 1$, we recover the results for a double-barrier junction of Refs. 17 and 18. In the limit $n \rightarrow \infty$, the shot-noise power approaches $\frac{1}{3} P_{\text {Poisson }}$ independent of $\Gamma$. By taking the continuum limit, $n \rightarrow \infty$, $\Gamma \rightarrow 1$, at fixed $n(1-\Gamma)$, we can study the shot noise in a diffusive conductor and the crossover to ballistic transport. We find exact agreement with a previous quantummechanical evaluation, ${ }^{8}$ in the limit of a conductance $\gg e^{2} / h$. It has been emphasized by Landauer, ${ }^{19}$ that Coulomb interactions may induce a further reduction of $P$. Here, we follow the quantum-mechanical treatments in assuming noninteracting electrons, leaving interaction effects for future work.

Let us begin by briefly reviewing the quantummechanical approach. The zero-temperature, zerofrequency shot-noise power $P$ of a phase-coherent conductor is related to the transmission matrix $t$ by the formula ${ }^{2,4}$

$$
P=P_{0} \operatorname{Tr} \underline{t} \underline{t}^{\dagger}\left(\underline{1}-\underline{t} \underline{t}^{\dagger}\right)=P_{0} \sum_{n=1}^{N} T_{n}\left(1-T_{n}\right),
$$

where $P_{0} \equiv 2 e|V| G_{0}$, with $V$ the applied voltage and $G_{0} \equiv e^{2} / h$ the conductance quantum (we assume spinless electrons for simplicity of notation), $T_{n} \in[0,1]$ an eigenvalue of $t t^{\dagger}$, and $N$ the number of transverse modes at the Fermi energy $E_{F}$. The conductarice is given by the Landauer formula

$$
G=G_{0} \operatorname{Tr} \underline{t}^{\dagger}=G_{0} \sum_{n=1}^{N} T_{n}
$$

One finds $P=2 e|V| G=P_{\text {Poisson }}$ for a conductor, where all $T_{n} \ll 1$ (such as a high tunnel barrier). However, if some $T_{n}$ are near 1 (open channels), then the shot noise is reduced below $P$ Poisson. In a phase-coherent metallic, 
diffusive conductor the $T_{n}$ are either exponentially small or of order unity. ${ }^{12}$ This bimodal distribution gives rise to the one-third suppression of the shot noise. ${ }^{6}$

We now formulate the semiclassical kinetic theory. ${ }^{13,14}$ We consider a $d$-dimensional conductor connected by ideal leads to two electron reservoirs. The fluctuating distribution function $f(\mathbf{r}, \mathbf{k}, t)$ equals $(2 \pi)^{d}$ times the density of electrons with position $\mathbf{r}$, and wave vector $\mathbf{k}$, at time $t$. [The factor $(2 \pi)^{d}$ is introduced so that $f$ is the occupation number of a unit cell in phase space.] The average over time-dependent fluctuations $\langle f\rangle \equiv \vec{f}$ obeys the Boltzmann equation,

$$
\begin{aligned}
& (d / d t+\mathcal{S}) \bar{f}(\mathbf{r}, \mathbf{k}, t)=0 \\
& \frac{d}{d t} \equiv \frac{\partial}{\partial t}+\mathbf{v} \cdot \frac{\partial}{\partial \mathbf{r}}+\mathcal{F} \cdot \frac{\partial}{\hbar \partial \mathbf{k}} .
\end{aligned}
$$

The derivative ( $3 \mathrm{~b}$ ) (with $\mathbf{v}=\hbar \mathbf{k} / m$ ) describes the classical motion in the force field $\mathcal{F}(\mathbf{r})$. The term $\mathcal{S} \bar{f}$ accounts for the stochastic effects of scattering. Only elastic scattering is taken into account and electron-electron scattering is disregarded. We consider the stationary situation, where $\bar{f}$ is independent of $t$. The time-dependent fluctuations $\delta f \equiv f-\bar{f}$ satisfy the Boltzmann-Langevin equation, ${ }^{13,14}$

$$
(d / d t+\mathcal{S}) \delta f(\mathbf{r}, \mathbf{k}, t)=j(\mathbf{r}, \mathbf{k}, t),
$$

where $j$ is a fluctuating source term. In the Boltzmann equation (3), scattering occurs into all wave vectors $\mathbf{k}$ with some probability distribution. Equation (4) takes into account that each electron is scattered into only one particular k. This implies that the flux $j$ is positive for that $\mathbf{k}$ and negative for the others. The flux $j$ has zero average, $\langle j\rangle=0$, and covariance

$$
\begin{aligned}
& \left\langle j(\mathbf{r}, \mathbf{k}, t) j\left(\mathbf{r}^{\prime}, \mathbf{k}^{\prime}, t^{\prime}\right)\right\rangle \\
& =(2 \pi)^{d} \delta\left(\mathbf{r}-\mathbf{r}^{\prime}\right) \delta\left(t-t^{\prime}\right) J\left(\mathbf{r}, \mathbf{k}, \mathbf{k}^{\prime}\right) .
\end{aligned}
$$

The $\delta$ functions ensure that fluxes are only correlated if they are induced by the same scattering process. The correlator $J$ depends on the type of scattering and on $\bar{f}$, but not on $\delta f$. The $J$ for impurity scattering is given in Ref. 14, and for barrier scattering it is given below.

We evaluate the fluctuating current $\delta I(t) \equiv I(t)-\bar{I}$ through cross section $S_{r}$ in the right lead,

$$
\delta I(t)=\frac{e}{(2 \pi)^{d}} \int_{S_{r}} d \mathbf{y} \int d \mathbf{k} v_{x} \delta f(\mathbf{r}, \mathbf{k}, t)
$$

Here, $\mathbf{r}=(x, \mathbf{y})$, with the $x$ coordinate along and $\mathbf{y}$ perpendicular to the lead. The zero-frequency noise power is given by

$$
P \equiv 2 \int_{-\infty}^{\infty} d t\langle\delta I(t) \delta I(0)\rangle
$$

The formal solution of Eq. (4) is

$$
\begin{aligned}
\delta f(\mathbf{r}, \mathbf{k}, t)= & \int_{-\infty}^{t} d t^{\prime} \int d \mathbf{r}^{\prime} \int d \mathbf{k}^{\prime} \\
& \times \mathcal{G}\left(\mathbf{r}, \mathbf{k} ; \mathbf{r}^{\prime}, \mathbf{k}^{\prime} ; t-t^{\prime}\right) j\left(\mathbf{r}^{\prime}, \mathbf{k}^{\prime}, t^{\prime}\right)
\end{aligned}
$$

where the Green's function $\mathcal{G}$ is a solution of

$$
(d / d t+\mathcal{S}) \mathcal{G}\left(\mathbf{r}, \mathbf{k} ; \mathbf{r}^{\prime}, \mathbf{k}^{\prime} ; t\right)=\delta\left(\mathbf{r}-\mathbf{r}^{\prime}\right) \delta\left(\mathbf{k}-\mathbf{k}^{\prime}\right) \delta(t)
$$

such that $\mathcal{G}=0$ if $t<0$. The transmission probability $T(\mathbf{r}, \mathbf{k})$ is the probability that an electron at $(\mathbf{r}, \mathbf{k})$ leaves the wire through the right lead. It is related to $\mathcal{G}$ by

$$
T(\mathbf{r}, \mathbf{k})=\int_{0}^{\infty} d t \int_{S_{\mathbf{r}}} d \mathbf{y}^{\prime} \int d \mathbf{k}^{\prime} v_{x}^{\prime} \mathcal{G}\left(\mathbf{r}^{\prime}, \mathbf{k}^{\prime} ; \mathbf{r}, \mathbf{k} ; t\right) .
$$

Equations (5)-(10) yield for the noise power the expression

$$
P=\frac{2 e^{2}}{(2 \pi)^{d}} \int d \mathbf{r} \int d \mathbf{k} \int d \mathbf{k}^{\prime} T(\mathbf{r}, \mathbf{k}) T\left(\mathbf{r}, \mathbf{k}^{\prime}\right) J\left(\mathbf{r}, \mathbf{k}, \mathbf{k}^{\prime}\right)
$$

Equation (11) applies generally to any conductor in which only elastic scattering occurs. We now specialize to the case that the scattering is due to $n$ planar tunnel barriers, perpendicular to the $x$ direction (see the inset of Fig. 1). Barrier $i$ has tunnel probability $\Gamma_{i} \in[0,1]$, which for simplicity is assumed to be $\mathbf{k}$ and $\mathbf{y}$ independent. Upon transmission $\mathbf{k}$ is conserved, whereas upon reflection $\mathbf{k} \rightarrow \widetilde{\mathbf{k}} \equiv\left(-k_{x}, \mathbf{k}_{y}\right)$. In what follows, we drop the (irrelevant) transverse coordinate $y$. At barrier $i$ (at $\left.x=x_{i}\right)$, the average densities $\bar{f}$ on the left side $\left(x_{i_{-}}\right)$and on the right side $\left(x_{i+}\right)$ are related by

$$
\bar{f}\left(x_{i+}, \mathbf{k}\right)=\Gamma_{i} \bar{f}\left(x_{i-}, \mathbf{k}\right)+\left(\mathbf{1}-\Gamma_{i}\right) \bar{f}\left(x_{i+}, \widetilde{\mathbf{k}}\right),
$$

for $k_{x}>0$. The relation for $k_{x}<0$ is Eq. (12), with $x_{i-}$ and $x_{i+}$ interchanged. To determine the correlator

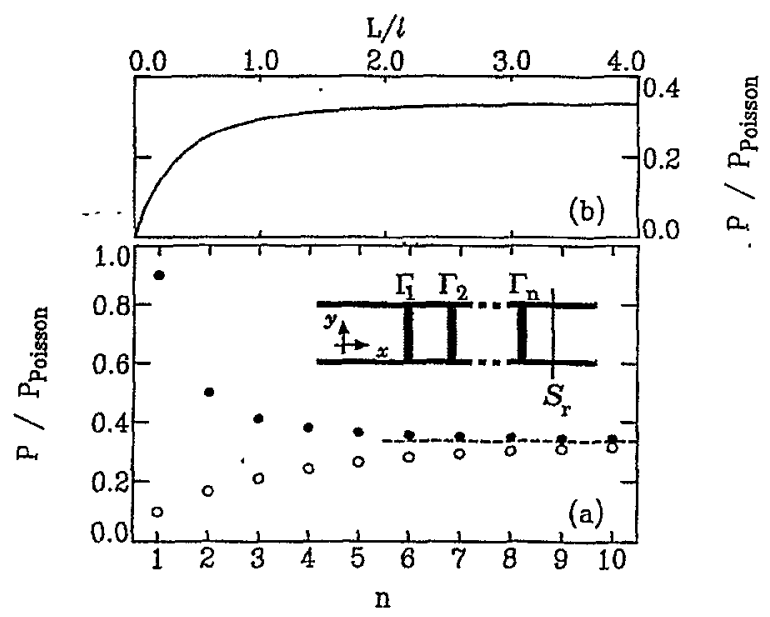

FIG. 1. (a) The shot-noise power $P$ for $n$ tunnel barriers in series with transmission probability $\Gamma=0.1$ (dots) and $\Gamma=0.9$ (circles), computed from Eq. (19). The dashed line is the large- $n$ limit $P=\frac{1}{3} P_{\text {Poisson }}$. The inset shows schematically the geometry considered. (b) The shot-noise power $P$ of a disordered wire, as a function of the ratio of length $L$ to mean free path $\ell$, according to Eq. (20). 
$J$ in Eq. (5), we argue in a similar way as in Ref. 5. Consider an incoming state from the left $\left(x_{i-}, \mathbf{k}\right)$ and from the right $\left(x_{i+}, \widetilde{\mathbf{k}}\right)$ (we assume $k_{\mathfrak{z}}>0$ ). If both incoming states are either filled or empty, there will be no fluctuations in the outgoing states, hence $\jmath=0$. Let us, therefore, consider the case that the incoming state from the left is filled and that from the right is empty, which occurs with probability $\bar{f}\left(x_{i-}, \mathbf{k}\right)\left[1-\bar{f}\left(x_{i+}, \widetilde{\mathbf{k}}\right)\right]$. On the average, the outgoing states at the left and right have occupation $1-\Gamma_{i}$ and $\Gamma_{i}$, respectively. However, since the incoming electron is either transmitted or reflected, the instantaneous occupation of the outgoing states differs from the average occupation. Upon transmission, the state at the right (left) has an excess (deficit) occupation of $1-\Gamma_{i}$. Transmission occurs with probability $\Gamma_{i}$, so the contribution to $J\left(x, \mathbf{k}, \mathbf{k}^{\prime}\right)$ from a transmitted electron is $\Gamma_{i}\left(1-\Gamma_{i}\right)^{2}\left[\delta\left(\mathbf{k}-\mathbf{k}^{\prime}\right)-\delta\left(\mathbf{k}-\tilde{\mathbf{k}}^{\prime}\right)\right] \delta\left(\boldsymbol{x}-x_{i}\right)\left|v_{x}\right|$. Similarly, a reflected electron contributes $\left(1-\Gamma_{i}\right) \Gamma_{i}^{2}\left[\delta\left(\mathbf{k}-\mathbf{k}^{\prime}\right)-\delta(\mathbf{k}-\right.$ $\left.\left.\widetilde{\mathbf{k}}^{\prime}\right)\right] \delta\left(x-x_{i}\right)\left|v_{x}\right|$. Collecting results, we find for $k_{x}>0$,

$$
\begin{aligned}
J\left(x, \mathbf{k}, \mathbf{k}^{\prime}\right)= & \sum_{i=\mathbf{1}}^{n} \delta\left(x-x_{i}\right) \Gamma_{i}\left(1-\Gamma_{i}\right) \\
& \times\left|v_{x}\right|\left[\delta\left(\mathbf{k}-\mathbf{k}^{\prime}\right)-\delta\left(\mathbf{k}-\widetilde{\mathbf{k}}^{\prime}\right)\right] \\
& \times\left\{\bar{f}\left(x_{i-}, \mathbf{k}\right)\left[1-\bar{f}\left(x_{i+}, \widetilde{\mathbf{k}}\right)\right]\right. \\
& \left.+\vec{f}\left(x_{i+}, \widetilde{\mathbf{k}}\right)\left[1-\bar{f}\left(x_{i-}, \mathbf{k}\right)\right]\right\}
\end{aligned}
$$

For $k_{x}<0$, Eq. (13) holds upon interchanging $x_{i-}$ and $x_{i+}$.

The average distribution function $\bar{f}$ inside the conductor depends on the equilibrium distributions $f_{l}$ and $f_{r}$ in the left and right reservoirs, according to

$$
\vec{f}(x, \mathbf{k})=T(x,-\mathbf{k}) f_{r}(\varepsilon)+[\mathbf{1}-T(x,-\mathbf{k})] f_{l}(\varepsilon),
$$

where $\varepsilon$ is the electron energy and $T(x,-\mathbf{k})$ equals the probability that an electron at $(x, \mathbf{k})$ has arrived there from the right reservoir. At zero temperature, one has $f_{l}(\varepsilon)=\Theta\left(E_{F}+e V-\varepsilon\right), f_{r}(\varepsilon)=\Theta\left(E_{F}-\varepsilon\right)$, with $\Theta$ the unit step function. Substitution of Eqs. (13) and (14) into Eq. (11) and linearization in $V$ yields

$$
\begin{aligned}
P= & P_{0} N \sum_{i=1}^{n} \Gamma_{i}\left(1-\Gamma_{i}\right) \\
& \times\left(T_{i}^{\rightarrow}-T_{i}^{\leftarrow}\right)^{2}\left(T_{i}^{\rightarrow}+T_{i}^{\leftarrow}-2 T_{i}^{\rightarrow} T_{i}^{\leftarrow}\right),
\end{aligned}
$$

where $T_{i}^{-} \equiv T\left(x_{i+}, k_{x}>0\right)\left[T_{i}^{\leftarrow} \equiv T\left(x_{2-}, k_{x}<0\right)\right]$ is the transmission probability into the right reservoir of an electron moving away from the right (left) side of barrier $i$. The conductance is given simply by

$$
G=G_{0} N T_{0}
$$

where $T_{0} \equiv T\left(x_{1-}, k_{x}>0\right)$ is the transmission probability through the whole conductor. Comparing Eqs. (2) and (16), we note that $\sum T_{n}$ corresponds semiclassically to $N T_{0}$. Comparison of Eqs. (1) and (15) shows that the semiclassical correspondence to $\sum_{n} T_{n}\left(1-T_{n}\right)$ is much more complicated, as it involves the transmission probabilities $T_{i}^{\rightarrow}, T_{i}^{+}$at all scatterers inside the conductor (and not just the transmission probability $T_{0}$ through the whole conductor).
As a first application of Eq. (15), we calculate the shot noise for a single tunnel barrier. Using $T_{0}=\Gamma$, $T_{1}^{\leftarrow}=0, T_{1}^{\rightarrow}=1$, we find the expected result ${ }^{1-5}$ $P=P_{0} N \Gamma(1-\Gamma)=(1-\Gamma) P_{\text {Poisson. }}$. The doublebarrier case $(n=2)$ is less trivial. Experiments by $L i$ et al., ${ }^{20}$ showed full Poisson noise for asymmetric structures $\left(\Gamma_{1} \ll \Gamma_{2}\right)$ and a suppression by one-half for the symmetric case $\left(\Gamma_{1} \simeq \Gamma_{2}\right)$. This effect has been explained by Chen and Ting, ${ }^{17}$ by Davies et al., ${ }^{18}$ and by others. ${ }^{21}$ These theories assume resonant tunneling in the regime that the applied voltage $V$ is much greater than the width of the resonance. This requires $\Gamma_{1}, \Gamma_{2} \ll 1$. The present semiclassical approach makes no reference to transmission resonances and is valid for all $\Gamma_{1}, \Gamma_{2}$. For the double-barrier system one has $T_{0}=\Gamma_{1} \Gamma_{2} / \Delta$, $T_{1}^{\leftarrow}=0, T_{1}^{\rightarrow}=\Gamma_{2} / \Delta, T_{2}^{\leftarrow}=\left(1-\Gamma_{1}\right) \Gamma_{2} / \Delta$, and $T_{2}^{\rightarrow}=1$, with $\Delta=\Gamma_{1}+\Gamma_{2}-\Gamma_{1} \Gamma_{2}$. From Eqs. (15) and (16), it follows that

$$
P=\frac{\Gamma_{1}^{2}\left(1-\Gamma_{2}\right)+\Gamma_{2}^{2}\left(1-\Gamma_{1}\right)}{\left(\Gamma_{1}+\Gamma_{2}-\Gamma_{1} \Gamma_{2}\right)^{2}} P_{\text {Poisson }} .
$$

In the limit $\Gamma_{1}, \Gamma_{2} \ll 1 \mathrm{Eq}$. (17) coincides precisely with the results of Refs. 17 and 18 .

The shot-noise suppression of one-half for a symmetric double-barrier junction has the same origin as the one-third suppression for a diffusive conductor. In our semiclassical model, this is evident from the fact that a diffusive conductor is the continuum limit of a series of tunnel barriers. We demonstrate this below. Quantum mechanically, the common origin is the bimodal distribution $\rho(T) \equiv\left\langle\sum_{n} \delta\left(T-T_{n}\right)\right\rangle$ of transmission eigenvalues, which for a double-barrier junction is given by ${ }^{22}$

$$
\rho(T)=\frac{N \Gamma_{1} \Gamma_{2}}{\pi T \sqrt{4 \Gamma_{1} \Gamma_{2} T-\left(\Delta T+\Gamma_{1} \Gamma_{2}\right)^{2}}}
$$

for $T \in\left[T_{-}, T_{+}\right]$, with $T_{ \pm}=\Gamma_{1} \Gamma_{2} /(1 \mp \sqrt{1-\Delta})^{2}$. For a symmetric junction $\left(\Gamma_{1}=\Gamma_{2} \ll 1\right)$, the density (18) is strongly peaked near $T=0$ and $T=1$, leading to a suppression of shot noise, just as in the case of a diffusive conductor. In fact, one can verify that the average of Eqs. (1) and (2), with the bimodal distribution (18), gives precisely the result (17) from the Boltzmann-Langevin equation.

We now consider $n$ barriers with equal $\Gamma$. We find $T_{0}=$ $\Gamma / \Delta, T_{i}^{\rightarrow}=[\Gamma+i(1-\Gamma)] / \Delta$, and $T_{i}^{+}=(i-1)(1-\Gamma) / \Delta$, with $\Delta=\Gamma+n(1-\Gamma)$. Substitution into Eqs. (15) and (16) yields

$$
P=\frac{1}{3}\left(1+\frac{n(1-\Gamma)^{2}(2+\Gamma)-\Gamma^{3}}{[\Gamma+n(1-\Gamma)]^{3}}\right) P_{\text {Poisson }} .
$$

The shot-noise suppression for a low barrier $(\Gamma=\overline{0} .9)$ and for a high barrier $(\Gamma=0.1)$ is plotted against $n$ in Fig. 1(a). For $\Gamma=0.1$, we observe almost full shot noise if $n=1$, one-half suppression if $n=2$, and on increasing $n$ the suppression rapidly reaches one-third. For $\Gamma=0.9$, we observe that $P / P_{\text {Poisson }}$ increases from almost zero to one-third. It is clear from Eq. (19) that $P \rightarrow \frac{1}{3} P_{\text {Poisson }}$ for $n \rightarrow \infty$ independent of $\Gamma$. 
Finally, we make the connection with elastic impurity scattering in a disordered wire. Here, the scattering occurs throughout the whole wire instead of at a discrete number of barriers. We have previously carried out a quantum-mechanical study of the shot noise in such a wire, ${ }^{8}$ on the basis of the Dorokhov-MelloPereyra-Kumar equation. ${ }^{23}$ For the semiclassical evaluation we take the limit $n \rightarrow \infty$ and $\Gamma \rightarrow 1$, such that $n(1-\Gamma)=L / \ell$. From Eq. (16), one then obtains the conductance $G=G_{0} N(1+L / \ell)^{-1}$. For the shot-noise power we find from Eq. (19),

$$
P=\frac{1}{3}\left[1-(1+L / \ell)^{-3}\right] P_{\text {Poisson }}
$$

This is precisely the result of Ref. 8 for a metallic wire $(N \ell / L \gg 1)$. Equation (20) is plotted in Fig. 1(b) and describes how the shot noise crosses over from com- plete suppression in the ballistic regime to one-third of the Poisson noise in the diffusive regime. The diffusive limit confirms Nagaev's calculation. ${ }^{7}$ Quantum corrections (of order $P_{0}$ ) to the shot-noise power, due to weak localization, ${ }^{8}$ cannot be obtained within our semiclassical approach.

In summary, we have presented a general framework to derive the shot noise from the semiclassical BoltzmannLangevin equation, and applied this to the case of conduction through a sequence of tunnel barriers. We obtain a sub-Poissonian shot-noise power, in complete agreement with quantum-mechanical calculations in the literature. This establishes that phase coherence is not required for the occurrence of suppressed shot noise in mesoscopic conductors.

This research was supported by the Dutch Science Foundation NWO/FOM.
${ }^{1}$ V. A. Khlus, Zh. Eksp. Teor. Fiz. 93, 2179 (1987) [Sov. Phys. JETP 66, 1243 (1987)].

${ }^{2}$ G. B. Lesovik, Pis'ma Zh. Eksp. Teor. Fiz. 49, 513 (1989) [JETP Lett. 49, 592 (1989)].

3 B. Yurke and G. P. Kochanski, Phys. Rev. B 41, 8184 (1990).

4 M. Büttiker, Phys. Rev. Lett. 65, 2901 (1990); Phys. Rev. B 46, 12485 (1992).

${ }^{5}$ Th. Martin and R. Landauer, Phys. Rev. B 45, 1742 (1992).

${ }^{6}$ C. W. J. Beenakker and M. Büttiker, Phys. Rev. B 46, 1889 (1992).

${ }^{7}$ K. E. Nagaev, Phys. Lett. A 169, 103 (1992).

${ }^{8}$ M. J. M. de Jong and C. W. J. Beenakker, Phys. Rev. B 46, 13400 (1992)

${ }^{9}$ Yu. V. Nazarov, Phys. Rev. Lett. 73, 134 (1994).

${ }^{10}$ B. L. Altshuler, L. S. Levitov, and A. Yu. Yakovets, Pis'ma Zh. Eksp. Teor. Fiz. 59, 821 (1994) [JETP Lett. 59, 857 (1994)].

${ }^{11}$ F. Liefrink, J. I. Dijkhuis, M. J. M. de Jong, L. W. Molenkamp, and $H$. van Houten, Phys. Rev. B 49, 14066 (1994).

12 O. N. Dorokhov, Solid State Commun. 51, 381 (1984); Y. Imry, Europhys. Lett. 1, 249 (1986); J. B. Pendry, A. MacKinnon, and P. J. Roberts, Proc. R. Soc. London Ser. A 437, 67 (1992).

${ }^{13}$ B. B. Kadomtsev, $\bar{Z}$ h. Eksp. Teor. Fiz. 32, 943 (1957) [Sov.
Phys. JETP 5, 771 (1957)].

${ }^{14}$ Sh. M. Kogan and A. Ya. Shul'man, Zh. Eksp. Teor. Fiz. 56, 862 (1969) [Sov. Phys. JETP 29, 467 (1969)].

${ }^{15}$ I. O. Kulik and A. N. Omel'yanchuk, Fiz. Nizk. Temp. 10, 305 (1984) [Sov. J. Low Temp. Phys. 10, 158 (1984)].

${ }^{18}$ C. W. J. Beenakker and H. van Houten, Phys. Rev. B 43, 12066 (1991). The results presented in this paper are incorrect, because the equal time correlation [Eq. (6) of Ref. $16]$ is not generally valid.

${ }^{17}$ L. Y. Chen and C. S. Ting, Phys. Rev. B 43, 4534 (1991).

18 J. H. Davies, P. Hyldgaard, S. Hershfield, and J. W. Wilkins, Phys. Rev. B 46, 9620 (1992).

${ }^{19}$ R. Landauer, in Fundamental Problems in Quantum Theory, edited by D. Greenberger (Academic Science, New York, to be published).

${ }^{20}$ Y. P. Li, A. Zaslavsky, D. C. Tsui, M. Santos, and M. Shayegan, Phys. Rev. B 41, 8388 (1990).

${ }^{21}$ S. Hershfield, J. H. Davies, P. Hyldgaard, C. J. Stanton, and J. W. Wilkins, Phys. Rev. B 47, 1967 (1992); K.-M. Hung and G. Y. Wu, ibid. 48, 14687 (1993); U. Hanke, Yu. M. Galperin, K. A. Chao, and N. Zou, ibid. 48, 17209 (1993).

22 J. A. Melsen and C. W. J. Beenakker, Physica B 203, 219 (1994).

23 O. N. Dorokhov, Pis'ma Zh. Eksp. Teor. Fiz. 36, 259 (1982) [JETP Lett. 36, 318 (1982)]; P. A. Mello, P. Pereyra, and N. Kumar, Ann. Phys. 181, 290 (1988). 